\title{
Cognitive function and 10 year mortality in an 85 year-old community-dwelling population
}

\author{
This article was published in the following Dove Press journal: \\ Clinical Interventions in Aging \\ 7 October 2014 \\ Number of times this article has been viewed
}

\author{
Yutaka Takata' \\ Toshihiro Ansai ${ }^{2}$ \\ Inho Soh ${ }^{2}$ \\ Shuji Awano ${ }^{2}$ \\ Ikuo Nakamichi' \\ Sumio Akifusa ${ }^{3}$ \\ Kenichi Goto' \\ Akihiro Yoshida ${ }^{2}$ \\ Hiroki Fujii' \\ Ritsuko Fujisawa' \\ Kazuo Sonoki ${ }^{3}$ \\ 'Division of General Internal \\ Medicine, Kyushu Dental University, \\ ${ }^{2}$ Division of Community Oral \\ Health Development, Kyushu Dental \\ University, ${ }^{3}$ Department of Oral \\ Health and Environment, School \\ of Oral Health Science, Kyushu Dental \\ University, Kitakyushu, Japan
}

Correspondence: Yutaka Takata Division of General Internal Medicine, Kyushu Dental University, Manazuru

2-6-I, Kokurakita-ku, Kitakyushu 803 8580, Japan

Tel +8I 93582 II3। (ext 20II)

Fax +8I 935820592

Email yutaka@kyu-dent.ac.jp

\begin{abstract}
The relationship between mortality and impaired cognitive function has not been thoroughly investigated in a very elderly community-dwelling population, and little is known about the association of disease-specific mortality with Mini-Mental State Examination (MMSE) subscale scores. Here we evaluated these data in Japanese community-dwelling elderly. In 2003, 85 year-olds ( $\mathrm{n}=207)$ were enrolled; 205 completed the MMSE for cognitive function and were followed-up for 10 years, during which time 120 participants died, 70 survived, and 17 were lost to follow-up. Thirty-eight deaths were due to cardiovascular disease, 22 to senility, 21 to respiratory disease, and 16 to cancer. All-cause mortality decreased by $4.3 \%$ with a 1 -point increase in the global MMSE score without adjustment, and it decreased by $6.3 \%$ with adjustment for both sex and length of education. Cardiovascular mortality decreased by $7.6 \%$ and senility mortality decreased by $9.2 \%$ with a 1 -point increase in the global MMSE score with adjustment for sex and education. No association was found between respiratory diseases or cancer mortality and global MMSE score. All-cause mortality also decreased with increases in MMSE subscale scores for time orientation, place orientation, delayed recall, naming objects, and listening and obeying. Cardiovascular mortality was also associated with the MMSE subscale of naming objects, and senility mortality was associated with the subscales of time orientation and place orientation. Thus, we found that impaired cognitive function determined by global MMSE score and some MMSE subscale scores were independent predictors of all-cause mortality or mortality due to cardiovascular disease or senility in 85 year-olds.
\end{abstract}

Keywords: cognition, elderly, mortality, community-dwelling, MMSE

\section{Introduction}

A systematic review of the literature regarding community-dwelling individuals aged 65 and over showed that the levels of cognitive impairment among these individuals were associated with an increased risk of mortality, and this association was found even for mild levels of impairment. ${ }^{1}$ A review focusing on the association of mild cognitive impairment with mortality, which included studies on population-based samples with persons aged 65 and over, found that the relative risk for participants with mild cognitive impairment varied from 1.0 to $1.9 .^{2}$ Another review article focusing on the association between geriatric syndrome and survival found that elderly persons aged 65 to 74 with advanced dementia have a higher risk of mortality than elderly persons without dementia, but the difference between people with and without dementia in remaining life expectancy may be 1 year or less in the "old-old", particularly 90 and over. ${ }^{3}$

In a large multicenter study of a population aged 65 years and over, an association between cognition and survival was relatively uniform across three age groups ( $<74$ years, 75-84 years, and $85+$ years), but no association was found in men 
aged $85+$, because of the reduced life expectancy in that age group. ${ }^{4}$ In a population of nursing home residents with the mean age of 83.8 years, individuals with both severe frailty syndrome and severe cognitive impairment were less likely to survive, but neither frailty nor cognitive impairment when considered separately was associated with a higher mortality rate. ${ }^{5}$ These investigations thus suggest that there is an association between cognitive impairment and high mortality in the elderly, but this association seems weaker in very elderly individuals (aged 80+ years).

Therefore, it is important to determine whether the association between cognitive function and mortality is still present in very elderly persons living in the community. To clarify this association in the very elderly, we conducted the present study to examine this issue in a community-dwelling population of 85 year-olds.

\section{Methods}

Our previous study ${ }^{6}$ was conducted with 827 participants who were 80 years old in 1998 (born in 1917) and resided in one of nine districts in Fukuoka Prefecture, Japan: Bunzen City, Munakata City, Yukuhashi City, Tobata Ward of Kitakyushu City, Kanda Town, Katsuyama Town, Toyotsu Town, Tsuiki Town, and Shinyoshitomi Village. There were 1,282 residents aged 80 years in these districts, and of these 827 agreed to participate in the 1998 study. Of the 827 individuals, 410 declined to participate in the present study and 210 died in the following 5 years; the 207 remaining 85 year-olds in 2003 (90 males, 117 females) participated in the present study. We compared sex and mortality differences between participants and non-participants in this study. Men were more prevalent in participants (43.2\%) than in non-participants $(35.6 \%, P=0.031)$, and mortality during 12 year follow-up from 80 years to 92 years was $39.3 \%$ for the former, and $68.8 \%$ for the latter $(P<0.001)$. However, this difference between these groups may be attributed partly to the fact that individuals who died before they reached the age of 85 years could not participate in the study started at 85 years. These findings indicate that participants used in the present study were not representative of the original study population in supplementary analyses, but the representatives of more healthy individuals.

Of the 207 participants, 205 (88 males, 117 females) completed the Japanese version of the Mini-Mental State Examination (MMSE) as an assessment of cognitive function, administered by an experienced speech/language/ hearing therapist or occupational therapist. The MMSE is a standardized evaluation of global cognitive function and is widely used in epidemiological studies. ${ }^{7-10}$ A full score on the MMSE is 30. The MMSE includes eleven test items: 1) time orientation (5 points); 2) place orientation (5 points); 3 ) registration of three words (3 points); 4) calculation: mentally subtracting seven iteratively from 100 (5 points); $5)$ delayed recall of the three words presented earlier (3 points); 6) naming objects (2 points); 7) repeating a sentence (1 point); 8) listening and obeying following a three-stage instruction (3 points); 9) reading and obeying following a message printed on a card (1 point); 10) writing a sentence (1 point), and 11) copying a figure on a sheet (1 point). One cognitive function expert participated in MMSE data collection. We did not assess interrater reliability. All of the present study's participants were also asked to fill out a medical questionnaire which included questions about current smoking, alcohol consumption, years of education, and history of stroke. After the baseline examination in 2003 , when all of the participants were 85 years old, the 207 participants were followed-up for 10 years. Confirmation of whether the individual was living or had died was obtained by asking the family via a telephone call or home visit. The causes of death were classified according to the tenth version of the International Classification of Diseases (ICD-10). ${ }^{11}$ The study was approved by the Human Investigations Committee of Kyushu Dental University, and was conducted in full accord with ethical principles, including the World Medical Association Declaration of Helsinki, as revised in 2002. Informed written consent was obtained from all participants in accordance with these principles.

All of the data are reported as means \pm standard deviation (SD). Participants were divided into three global MMSE groups; preserved function (global MMSE $\geq 27$ ), minimally impaired function (global MMSE 26 to 23), and moderately impaired function (global MMSE $\leq 22$ ). This MMSE grouping were based on a tertile split. To have a similar number of individuals for each group a cut-off MMSE value was used to divide the three groups.

The differences in mean values among the three global MMSE groups were examined using an analysis of variance (ANOVA) and those between two groups were evaluated using the unpaired Student's $t$-test. Unpaired Student's $t$-test was done between men and women, or survivor and non-survivor for comparing MMSE scores. The categorical variables were compared using the chi-squared test. The associations between the three global MMSE groups and 10 year mortality rates were assessed using multivariate Cox proportional hazards regression analyses in which no adjustment was made, or only adjustment for sex was made, both 
sex and education periods were adjusted, or hypertension, diabetes, heart disease, cancer, and diet were adjusted. Proportional hazards assumption using Schoenfeld residuals was not tested in the Cox models. A comparison of the survival rates among the three global MMSE groups was conducted by the Kaplan-Meier method, followed by a log-rank test to assess the significance between the survival curves. The results were considered to be significant at $P<0.05$. The significance threshold for multiple comparisons made by Cox proportional hazards regression analyses was adjusted using the Bonferroni method of adjusting for multiple comparisons, being changed from 0.05 to 0.0125 .

\section{Results}

During the 10 year follow-up period, of the 207 participants, 120 had died, 70 survived, and 17 were lost to follow-up. Of the 120 participants who had died, 38 deaths were due to cardiovascular disease, 22 to senility, 21 to respiratory tract diseases, 16 to cancers, four to extrinsic causes, three to gastrointestinal disease, two to urinary tract disease, two to other diseases, and 12 to unknown causes. ICD-10 codes were I00-I99 for cardiovascular disease, J00-J99 for respiratory disease, C00-D48 for cancer, and R54 for senility.

For the total study population, the average global MMSE score (ie, the total score for all eleven items) was 23.8 \pm 4.5 ; for the 90 men, $24.0 \pm 4.4$, and for the 117 women, 23.6 44.7 , a nonsignificant difference. The global MMSE score for the 70 survivors was $24.8 \pm 4.0$, which is significantly higher than the corresponding scores for the 120 non-survivors (23.1 \pm 4.8$)(P=0.012)$. The average education period was $9.4 \pm 2.6$ years for all subjects, 9.9 \pm 3.0 years for men, and $9.0 \pm 2.1$ years for women $(P=0.004)$. Education period was positively correlated with MMSE score $(r=0.259, P<0.001)$.
MMSE scores were 21.2 \pm 5.1 for individuals who had 6 years or less education (graduated only from elementary school, $\mathrm{n}=25$ ), 23.8 \pm 3.5 for those who had 7-9 years (graduated from junior high school and elementary school, $n=76$ ), $24.8 \pm 4.2$ for those who had 10-12 years (graduated from high school, junior high school, and elementary school, $\mathrm{n}=74$ ), and $25.3 \pm 2.8$ for those who had 13 years or more (graduated from university, high school, junior high school, and elementary school, $\mathrm{n}=20 ; P=0.001)$. MMSE scores were $24.8 \pm 4.0$ for individuals who survived until 95 years or older, $23.1 \pm 5.3$ for those who survived until 92-94 years, $24.2 \pm 4.0$ for those who survived until 90 or 91 years, and $22.4 \pm 4.8$ for those who survived until $85-89$ years or less $(P=0.029)$. There was a slight positive correlation between age of death and MMSE scores $(r=0.161, P=0.029)$.

We divided the participants into the following three groups based on their cognitive function as shown by their global MMSE scores so that similar numbers of participants were in each group: preserved function (global MMSE $\geq 27, n=60$ ), minimally impaired function (global MMSE 26-23, n=86), and moderately impaired function (global MMSE $\leq 22, n=59$ ). The baseline characteristics of each group are shown in Table 1. Total mortality was higher in the group with moderately impaired function and lower in the group with preserved function $(P=0.017)$. No significant difference was found in mortalities due to cardiovascular disease, cancer, respiratory disease, or senility. The level of education was higher in the preserved-function group and lower in the moderately-impaired group $(P<0.001)$. There were no significant differences in sex, smoking, alcohol consumption, and history of stroke.

Table 2 shows the results of the multivariate Cox analyses of the three cognition groups for total mortality without

Table I Baseline characteristics of the 205 elderly participants with different cognitive function levels grouped by MMSE scores

\begin{tabular}{|c|c|c|c|c|}
\hline & $\begin{array}{l}\text { Preserved function } \\
\text { (global MMSE } \geq 27 \text { ) }\end{array}$ & $\begin{array}{l}\text { Minimally impaired function } \\
\text { (global MMSE } 26 \text { to } 23 \text { ) }\end{array}$ & $\begin{array}{l}\text { Moderately impaired function } \\
\text { (global MMSE } \leq 22 \text { ) }\end{array}$ & P-value \\
\hline$n$ & 60 & 86 & 59 & \\
\hline Men & $28 / 60(46.7)$ & $30 / 86(34.9)$ & $30 / 59(50.8)$ & 0.127 \\
\hline Total mortality & $27 / 54(50.0)$ & $49 / 79(62.0)$ & $42 / 55(76.4)$ & 0.017 \\
\hline Cardiovascular death & $8 / 54(14.8)$ & I3/79 (16.5) & I7/55 (30.9) & 0.062 \\
\hline Cancer death & $4 / 54$ (7.4) & $6 / 79(7.6)$ & $5 / 55(9.1)$ & 0.936 \\
\hline Respiratory death & $7 / 54(13.0)$ & $7 / 79$ (8.9) & $7 / 55$ (12.7) & 0.693 \\
\hline Senility death & $2 / 54(3.7)$ & $12 / 79(15.2)$ & $7 / 55$ (12.7) & 0.108 \\
\hline Education (years) & $10.50 \pm 2.62$ & $9.04 \pm 2.15$ & $8.78 \pm 2.72$ & $<0.001$ \\
\hline History of stroke & $3 / 60(5.0)$ & $8 / 86(9.3)$ & $4 / 59(6.8)$ & 0.607 \\
\hline Smoking & $3 / 59(5.1)$ & $6 / 86(7.0)$ & $\mathrm{I} / 56(\mathrm{I} .8)$ & 0.380 \\
\hline Alcohol consumption & $35 / 58(60.3)$ & $42 / 84(50.0)$ & $26 / 57(45.6)$ & 0.262 \\
\hline
\end{tabular}

Note: Data are shown as $\mathrm{n}(\%)$ or mean \pm SD.

Abbreviations: MMSE, Mini-Mental State Examination; SD, standard deviation. 
adjustment (Table 2, Group A), with adjustment only for sex (Table 2, Group B), with adjustment for both sex and education (Table 2, Group C), and with adjustment for sex and education, histories of hypertension, diabetes, heart disease, cancer, and diet (Table 2, Group D). Without adjustment, the total mortality was 2.0 times higher in the moderately impaired group than in the preserved group, whereas no significant difference was found between the minimally impaired group and the preserved group. Similarly, with adjustment for sex, the moderately impaired group had 2.2 times higher mortality than the preserved group. The men were 2.1 times more likely to die than the women. With the adjustment for both sex and education, the total mortality was also 2.3 times higher in participants with moderately impaired cognitive function and 1.7 times higher in those with minimally impaired cognitive function compared to those with preserved cognitive function. Using Bonferroni method of adjusting for multiple comparisons, however, total mortality did not differ between the preserved group and the minimally impaired group. The moderately impaired group had 2.6 times higher total mortality, and the minimally impaired group had 1.9 times higher mortality than the preserved group when adjusted for sex and education, histories of hypertension, diabetes, heart disease, cancer, and diet. This difference between the preserved group and the minimally impaired group also disappeared with Bonferroni adjustment for multiple comparison.

We also assessed the associations of disease-specific mortality with the three cognition groups by multivariate Cox proportional hazards regression analyses. Without adjustment, the cardiovascular disease mortality was 2.8 times higher in the participants with moderately impaired cognitive function than in those with preserved cognition (hazard ration [HR] 2.78, 95\% confidence interval [CI] 1.198-6.457, $P=0.017$ ), and was 3.1 times higher in the former than the latter with adjustment for sex and education (HR 3.08, 95\% CI 1.274-7.438, $P=0.012$ ). Mortality due to senility was significantly higher in the minimally impaired group compared to the preserved group both without adjustment (HR 5.14, 95\% CI 1.150-22.980, $P=0.032$ ) and with adjustment for sex and education (HR 4.72, 95\% CI 1.028-21.628, $P=0.046$ ). These differences in disease-specific mortality disappeared after Bonferroni adjustment. There were no associations between mortality due to respiratory disease or cancer and the three cognition groups.

Table 2 Multivariate Cox analysis of the three cognition groups for total mortality

\begin{tabular}{|c|c|c|c|}
\hline & Hazard ratio & $95 \% \mathrm{Cl}$ & $P$-value \\
\hline \multicolumn{4}{|l|}{$\mathbf{A}$} \\
\hline Preserved group & I & & \\
\hline Minimally impaired group & 1.46 & $0.912-2.342$ & 0.115 \\
\hline Moderately impaired group & 2.05 & $1.259-3.334$ & 0.004 \\
\hline \multicolumn{4}{|l|}{ B } \\
\hline Preserved group & I & & \\
\hline Minimally impaired group & 1.60 & $0.996-2.57 \mid$ & 0.052 \\
\hline Moderately impaired group & 2.21 & $1.357-3.610$ & 0.001 \\
\hline Men & 2.11 & $1.457-3.045$ & 0.000 \\
\hline \multicolumn{4}{|l|}{ C } \\
\hline Preserved group & $\mathrm{I}$ & & \\
\hline Minimally impaired group & 1.70 & $1.024-2.820$ & 0.040 \\
\hline Moderately impaired group & 2.27 & $1.343-3.843$ & 0.002 \\
\hline Men & 1.94 & $1.328-2.839$ & 0.001 \\
\hline Education & 1.01 & $0.936-1.095$ & 0.755 \\
\hline \multicolumn{4}{|l|}{ D } \\
\hline Preserved group & I & & \\
\hline Minimally impaired group & 1.93 & $1.131-3.280$ & 0.016 \\
\hline Moderately impaired group & 2.65 & $|.505-4.65|$ & 0.001 \\
\hline Men & 2.06 & $1.378-3.074$ & 0.000 \\
\hline Education & 0.99 & $0.911-1.085$ & 0.892 \\
\hline Hypertension & 0.85 & $0.563-1.288$ & 0.447 \\
\hline Diabetes & 0.50 & $0.248-1.022$ & 0.057 \\
\hline Heart disease & 0.96 & $0.607-1.512$ & 0.854 \\
\hline Cancer & 0.15 & $0.051-0.439$ & 0.001 \\
\hline Diet & 1.05 & $0.574-1.917$ & 0.876 \\
\hline
\end{tabular}

Notes: Without adjustment (A), with adjustment for sex (B), with adjustment for sex and education (C), with adjustment for sex and education, histories of hypertension, diabetes, heart disease, cancer, and diet (D).

Abbreviation: $\mathrm{Cl}$, confidence interval. 
Multivariate Cox analyses were also assessed for 1-point increases in the participants' global MMSE scores for total mortality without adjustment, with adjustment only for sex, with adjustment for both sex and education, and with adjustment for sex and education, histories of hypertension, diabetes, heart disease, cancer, and diet. Total mortality decreased by $4.3 \%$ (HR $0.957, P=0.013$ ) with a 1 -point increase in MMSE score when no adjustment was made. With the adjustment for sex, total mortality decreased by $5.5 \%$ (HR 0.945 , $P=0.002)$ with a 1-point increase in MMSE score, but with the adjustment for both sex and education, the total mortality decreased by $6.3 \%$ (HR $0.937, P=0.004$ ) with a 1-point increase in MMSE score. Similarly, total mortality decreased by $6.8 \%$ (HR $0.932, P=0.003$ ) with a 1-point increase in MMSE with adjustment for sex and education, histories of hypertension, diabetes, heart disease, cancer, and diet.

We also conducted multivariate Cox analyses of 1-point differences in global MMSE scores for disease-specific mortality with and without adjustments for sex and education. Mortality from cardiovascular diseases decreased by $7.6 \%$ with a 1-point increase in global MMSE score when adjusted for both sex and education (HR 0.924, 95\% CI 0.858-0.995, $P=0.036$ ). Without adjustment, senility mortality decreased by $9.8 \%$ with a 1-point increase in global MMSE score (HR $0.912,95 \%$ CI $0.853-0.975, P=0.007$ ), and with adjustment for sex and education it decreased by $9.2 \%$ (HR $0.908,95 \% \mathrm{CI}$ $0.825-0.999, P=0.047)$. There was no significant association of mortalities due to respiratory diseases or cancers with a 1-point change in global MMSE score with or without adjustment.

The results of the multivariate Cox analyses of one MMSE subscale point for total mortality are shown in Table 3; without adjustment (Table 3, Group A), with adjustment only for sex (Table 3, Group B), with adjustment both for sex and education (Table 3, Group C), and with adjustment for sex and education, histories of hypertension, diabetes, heart disease, cancer, and diet (Table 3, Group D). With a 1-point increase in time orientation, the total mortality decreased by $15.5 \%$ when no adjustment was made; it decreased by $18.8 \%$ when an adjustment was made only for sex, it decreased by $18.9 \%$ when adjustments were made for sex and education, and it decreased by $21.1 \%$ with adjustment for sex and education, histories of hypertension, diabetes, heart disease, cancer, and diet. Similarly, with a 1-point increase in delayed recall of words, total mortality decreased by $19.7 \%$ without adjustment, $19.8 \%$ with sex adjustment, and $19.4 \%$ with adjustment for sex and education.

Slight associations between total mortality and MMSE subscale points were also found for naming objects (with no adjustment, with adjustment for both sex and education, and with adjustment for sex and education, histories of hypertension, diabetes, heart disease, cancer, and diet), place orientation (with adjustment for sex, and with adjustment for both sex and education), and listening and obeying (with adjustment for sex, and with adjustment for both sex and education, and with adjustment for sex and education, histories of hypertension, diabetes, heart disease, cancer, and diet). With Bonferroni adjustment for multiple comparison, significant associations between total mortality and one MMSE subscale point were only found for time orientation and delayed recall of words.

We also performed multivariate Cox analyses of one MMSE subscale point for disease-specific mortality without adjustment, with adjustment only for sex, and with adjustment both for sex and education. Mortality due to cardiovascular diseases decreased by $96.6 \%$ with a 1-point increase in the naming objects subscale score (HR 0.034, 95\% CI $0.004-0.286, P=0.002)$ when no adjustment was made; it decreased by $95.3 \%$ (HR $0.047,95 \%$ CI 0.006-0.397, $P=0.005$ ) when adjustment was made for sex, and it decreased by $96.7 \%$ (HR $0.033,95 \%$ CI $0.004-0.309$, $P=0.003$ ) when the adjustment was made for both sex and education. No association was found for other subscale scores with cardiovascular mortality.

Mortality due to senility decreased by $35.0 \%$ with a 1-point increase in the time orientation subscale score (HR $0.650,95 \%$ CI $0.527-0.802, P<0.001)$ when no adjustment was made; it decreased by $35.4 \%$ (HR $0.646,95 \% \mathrm{CI}$ $0.519-0.804, P<0.001$ ) when adjustment was made for sex, and it decreased by $35.2 \%$ (HR $0.648,95 \%$ CI $0.509-0.823$, $P<0.001)$ when the adjustment was made for both sex and education. Similarly, mortality due to senility decreased by $34.9 \%$ with a 1-point increase in the place orientation subscale score (HR 0.651, 95\% CI 0.475-0.891, $P=0.007$ ) when no adjustment was made, and it decreased by $34.9 \%$ (HR $0.651,95 \%$ CI $0.470-0.900, P=0.009$ ) when the adjustment was done for sex, and decreased by $37.9 \%$ (HR $0.621,95 \%$ CI $0.423-0.913, P=0.015$ ) when adjusted for both sex and education. No association was found for other subscale scores with senility mortality. Mortality due to respiratory disease or cancer was not associated with any MMSE subscale scores.

The survival curves of participants who survived during the 10 year follow-up period among the three cognition groups were determined for all participants (Figure 1A), the men (Figure 1B), and the women (Figure 1C). The average survival period was 95.9 months for the preserved cognition group, 84.8 months for the minimally impaired 
Table 3 Multivariate Cox analysis of one MMSE subscale point for total mortality

\begin{tabular}{|c|c|c|c|}
\hline & Hazard ratio & $95 \% \mathrm{Cl}$ & $P$-value \\
\hline \multicolumn{4}{|l|}{ A } \\
\hline Time orientation & 0.845 & $0.752-0.948$ & 0.004 \\
\hline Place orientation & 0.863 & $0.729-1.021$ & 0.087 \\
\hline Registration of words & 0.929 & $0.647-1.334$ & 0.690 \\
\hline Calculation & 0.952 & $0.850-1.066$ & 0.395 \\
\hline Delayed recall of words & 0.803 & $0.681-0.946$ & 0.009 \\
\hline Naming objects & 0.096 & $0.013-0.724$ & 0.023 \\
\hline Repeating sentence & 0.743 & $0.492-1.122$ & 0.158 \\
\hline Listening and obeying & 0.524 & $0.274-1.003$ & 0.051 \\
\hline Reading and obeying & 0.711 & $0.290-1.743$ & 0.456 \\
\hline Writing sentence & 0.893 & $0.519-1.537$ & 0.684 \\
\hline Copying figure & 0.884 & $0.588-1.328$ & 0.552 \\
\hline \multicolumn{4}{|l|}{ B } \\
\hline Time orientation & 0.812 & $0.721-0.914$ & 0.001 \\
\hline Place orientation & 0.807 & $0.681-0.957$ & 0.013 \\
\hline Registration of words & 0.976 & $0.678-1.405$ & 0.895 \\
\hline Calculation & 0.919 & $0.820-1.030$ & 0.145 \\
\hline Delayed recall of words & 0.802 & $0.680-0.946$ & 0.009 \\
\hline Naming objects & 0.134 & $0.018-1.014$ & 0.052 \\
\hline Repeating sentence & 0.770 & $0.510-1.164$ & 0.215 \\
\hline Listening and obeying & 0.426 & $0.220-0.825$ & 0.011 \\
\hline Reading and obeying & 0.518 & $0.207-1.296$ & 0.160 \\
\hline Writing sentence & 0.804 & $0.466-1.388$ & 0.434 \\
\hline Copying figure & 0.791 & $0.524-1.194$ & 0.265 \\
\hline \multicolumn{4}{|l|}{ C } \\
\hline Time orientation & $0.81 \mathrm{I}$ & $0.711-0.926$ & 0.002 \\
\hline Place orientation & 0.803 & $0.652-0.988$ & 0.038 \\
\hline Registration of words & 1.098 & $0.676-1.783$ & 0.706 \\
\hline Calculation & 0.935 & $0.829-1.054$ & 0.272 \\
\hline Delayed recall of words & 0.806 & $0.678-0.958$ & 0.015 \\
\hline Naming objects & 0.112 & $0.014-0.873$ & 0.037 \\
\hline Repeating sentence & 0.853 & $0.550-1.324$ & 0.479 \\
\hline Listening and obeying & 0.424 & $0.202-0.888$ & 0.023 \\
\hline Reading and obeying & 0.702 & $0.164-3.002$ & 0.634 \\
\hline Writing sentence & 0.852 & $0.457-1.59 \mid$ & 0.616 \\
\hline Copying figure & 0.814 & $0.526-1.259$ & 0.355 \\
\hline \multicolumn{4}{|l|}{ D } \\
\hline Time orientation & 0.799 & $0.700-0.912$ & 0.001 \\
\hline Place orientation & 0.845 & $0.676-1.057$ & 0.140 \\
\hline Registration of words & 1.539 & $0.915-2.589$ & 0.104 \\
\hline Calculation & 0.943 & $0.828-1.072$ & 0.369 \\
\hline Delayed recall of words & 0.849 & $0.701-1.029$ & 0.095 \\
\hline Naming objects & 0.087 & $0.011-0.696$ & 0.021 \\
\hline Repeating sentence & 0.783 & $0.496-1.236$ & 0.294 \\
\hline Listening and obeying & 0.423 & $0.190-0.937$ & 0.034 \\
\hline Reading and obeying & 0.713 & $0.160-3.171$ & 0.657 \\
\hline Writing sentence & 0.685 & $0.358-1.310$ & 0.253 \\
\hline Copying figure & 0.754 & $0.481-1.182$ & 0.219 \\
\hline
\end{tabular}

Notes: Without adjustment (A), with adjustment for sex (B), with adjustment for sex and education (C), with adjustment for sex and education, histories of hypertension, diabetes, heart disease, cancer, and diet (D).

Abbreviations: MMSE, Mini-Mental State Examination; $\mathrm{Cl}$, confidence interval.

group, and 77.5 months for the moderately impaired group. The cumulative survival rate was significantly lower in the moderately impaired group than in the preserved group $(P=0.003)$. There was no significant difference in survival rate between the moderately impaired group and the minimally impaired group. Among the 82 surviving men, the average survival period was 85.1 months for the preserved group, 77.4 months for the minimally impaired group, and 59.8 months for the moderately impaired group. The cumulative survival period was significantly shorter in 

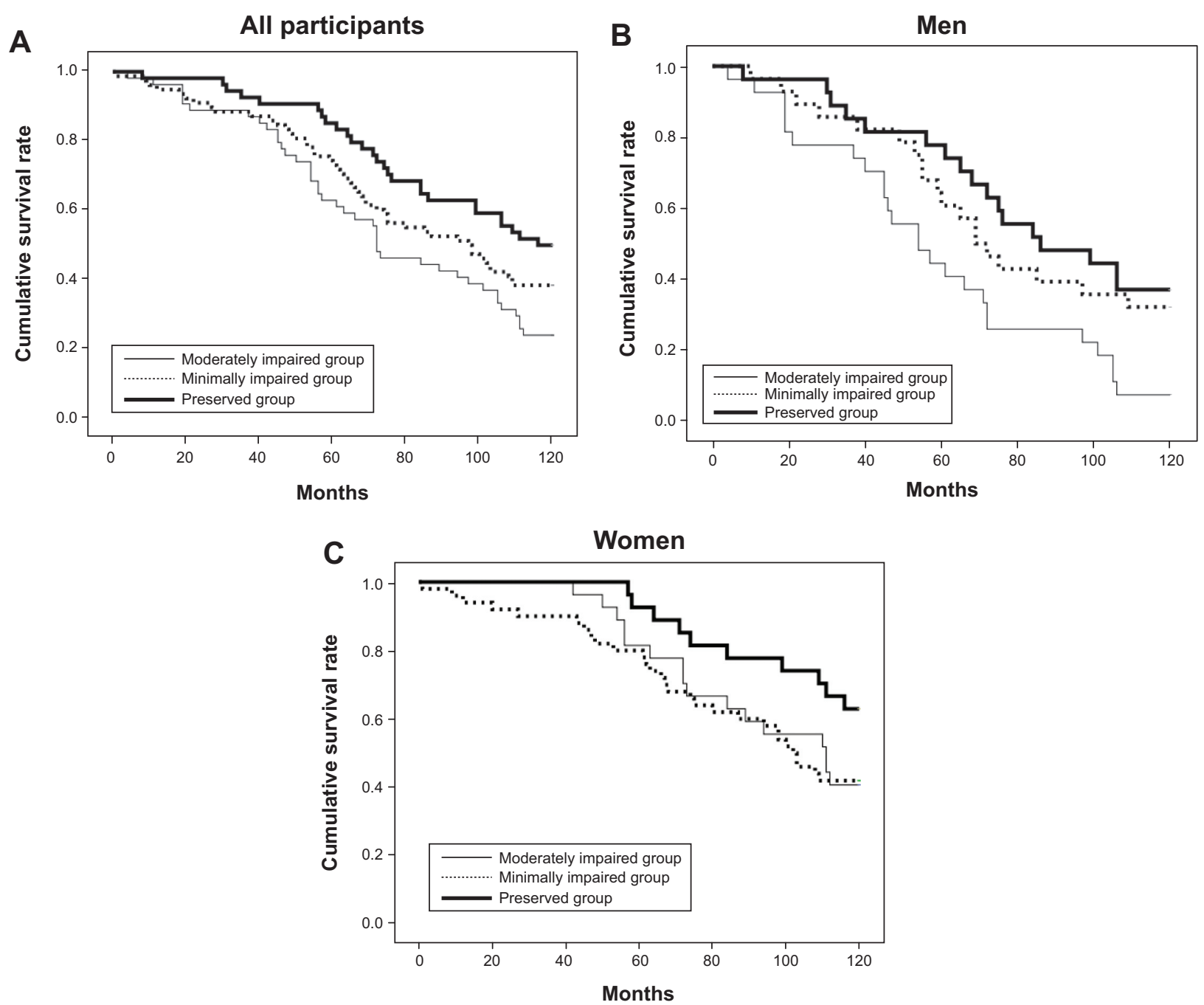

Figure I Survival curves of the 70 elderly participants who survived during the 10 year follow-up period among the three cognition groups.

Notes: All participants $(\mathbf{A})$, men $(\mathbf{B} ; n=82)$, and women $(\mathbf{C} ; n=104)$. The participants were divided into three groups: preserved group, global MMSE $\geq 27$, $n=54$; minimally impaired group global MMSE 26 to 23, $n=78$; moderately impaired group, global MMSE $\leq 22, n=54$.

Abbreviation: MMSE, Mini-Mental State Examination.

the moderately impaired group than in the preserved group $(P=0.004)$ and compared to the minimally impaired group $(P=0.029)$.

Among the 104 surviving women, the average survival period was 106.8 months for the preserved group, 88.9 months for the minimally impaired group, and 95.1 months for the moderately impaired group, with no significant difference in cumulative survival period among the three groups.

\section{Discussion}

In the present study, we observed a relationship between total mortality and impaired cognitive function in a very elderly population ( 85 year-olds). Total mortality was increased not only with a decrease in global MMSE scores but also with decreases in subscale scores of MMSE, ie, time orientation, delayed recall of words, naming objects, place orientation, and listening and obeying. Both senility-linked mortality and cardiovascular mortality increased with the decrease in global MMSE score, whereas mortalities due to respiratory disease or cancer were not associated with global MMSE scores. Similarly, mortality due to senility increased with decreases in MMSE subscale scores of time orientation and place orientation, and mortality due to cardiovascular disease increased with decreases in the MMSE subscale scores for naming objects. Mortality due to respiratory disease or cancer was not associated with any MMSE subscale scores.

The present findings that there were associations in cardiovascular mortality with global MMSE scores or MMSE subscale scores suggest that cerebrovascular diseases together with strokes related to impaired cognitive function may elevate total mortality and cardiovascular mortality in the very elderly. Associations between senility mortality and global MMSE 
scores or MMSE subscale scores suggest that senility induced by terminal status of cardiac failure, stroke, malnutrition, multiple organ failure or other serious diseases may impair cognitive function in 85 year-olds. Impaired cognitive function found by decreased scores of global MMSE or subscale MMSE may independently predict total mortality, cardiovascular mortality, and senility mortality in the community-dwelling very elderly population. A new finding of this study was that these associations were present in community-dwelling 85 year-olds.

In a very elderly population (95 year-olds) studied by Borjesson-Hanson et $\mathrm{al}^{12}$ cognitive function measured using the MMSE predicted mortality, and mortality decreased by $13 \%$ for each 1-point increase in the MMSE. Another study of a community-dwelling elderly population with the average age of 78.9 years showed that impaired cognitive function as assessed by the MMSE predicted mortality in men and women. ${ }^{13}$ A low MMSE score was the strongest predictor of mortality in homedwelling patients aged 75 years and older with cardiovascular disease.${ }^{14}$ Mild to moderate cognitive impairment was a major risk for mortality in patients with the mean age of 84 years with acute hip fracture. ${ }^{15}$ Fast cognitive decline at the time of dementia diagnosis in a community-based elderly population with a mean age of 78.8 years was a major prognostic factor for higher mortality rate. ${ }^{16}$ Similarly, dementia diagnosed after the age of 75 years was a predictor of mortality. ${ }^{17}$ These findings are in agreement with our finding that impaired cognitive function was associated with higher mortality in a communitydwelling population of 85 year-olds. Survival periods of people with dementia was 4.3 years for ages 80-89 and 3.8 years for ages 90 and older as estimated according to a population based cohort study with a 14 year follow-up period. ${ }^{18}$

O’Donnell et $\mathrm{al}^{8}$ reported that in people aged 55 years or over at increased cardiovascular disease risk, impairment of cognitive function as shown by the MMSE was associated with increases in the risks of stroke, congestive heart failure, and cardiovascular death. They also found associations of MMSE subscale scores for time orientation, place orientation, calculation, delayed recall, and copying with cardiovascular mortality. We also found, in a very elderly population, that not only all-cause mortality but also disease-specific mortalities due to cardiovascular diseases or senility were associated with impaired global cognitive function as determined by the MMSE. Mortality due to cardiovascular diseases also decreased with an increase in the subscale score for naming objects. Thus, our findings have extended the association between impaired global or subscale cognitive function and increased mortality due to cardiovascular disease, in a very elderly population of 85 year-olds.
In a study of community-dwelling Japanese aged 79 years and older, ${ }^{9}$ all-cause mortality increased with decreases in the scores of the MMSE subscales for time orientation, place orientation, calculation, delayed recall, repeating a sentence, and copying a figure. Similarly, in Koreans aged 60 and over, impaired cognitive function as determined by not only global MMSE score, but also the MMSE subscale scores for time orientation and calculation, was associated with increased total mortality. ${ }^{10}$ We also found in our population an association between total mortality with MMSE subscale scores for time orientation, recall of words, naming objects, place orientation, and listening and obeying, indicating that an association with all-cause mortality may be found for global MMSE scores or MMSE subscale scores even in 85 year-olds, a very elderly population.

However, there are limitations in the present study. The number of participants was not large. Many findings of no significant difference in this study are due to small sample size or small number of events. Seventeen of the 207 participants were also lost to follow-up during a 10 year period. The sample used in the present study were not representative of the original study population, but representative of more healthy individuals. There was also an issue of survivorship. Additionally, MMSE was reported to have poor reliability and sensitivity, ${ }^{19,20}$ and to be poor in discriminating individual cognitive difference in non-demented elderly subjects. ${ }^{21}$

In conclusion, we found that impaired cognitive function as determined by global MMSE scores and by some MMSE subscale scores was an independent predictor of total cause mortality and mortality due to cardiovascular disease or senility in a population of 85 year-olds.

\section{Acknowledgments}

This work was supported in part by Grants in Aid for Scientific Research (B) number 24390472 (Yutaka Takata) and number 21390560 (Yutaka Takata).

\section{Disclosure}

There are no conflicts of interest to report.

\section{References}

1. Dewey ME, Saz P. Dementia, cognitive impairment and mortality in persons aged 65 and over living in the community: a systematic review of the literature. Int J Geriatr Psychiatry. 2001;16(8):751-761.

2. Guehne U, Angermeyer MC, Riedel-Heller S. Is mortality increased in mildly cognitively impaired individuals? Dement Geriatr Cogn Disord. 2006;21(5-6):403-410.

3. Kane RL, Shamliyan T, Talley K, Pacala J. The association between geriatric syndrome and survival. J Am Geriatr Soc. 2012;60(5):896-904. 
4. Neale R, Brayne C, Johnson AL; Medical Research Council Cognitive Function and Ageing Study Writing Committee. Cognition and survival: an exploration in a large multicentre study of the population aged 65 years and over. Internat J Epidemiol. 2001;30(6):1383-1388.

5. Matsusik P, Tomaazewski K, Chmielowska K, et al. Severe frailty and cognitive impairment are related to higher mortality in 12-month follow-up of nursing home residents. Arch Gerontol Geriatr. 2012; 55(1):22-24.

6. Takata Y, Ansai T, Soh I, et al. Association between Body Mass Index and Mortality in an 80-Year-Old Population. J Am Geriatr Soc. 2007; 55(6):913-917.

7. Folstein MF, Folstein SE, McHugh PR. "Mini-Mental State." A practical method for grading the cognitive state of patients for the clinician. J Psychiatr Res. 1975;12(3):189-198.

8. O’Donnell M, Teo K, Gao P, et al. Cognitive impairment and risk of cardiovascular events and mortality. Eur Heart J. 2012;33(14):1777-1786.

9. Iwasa H, Kai I, Yoshida Y, Suzuki T, Kim H, Yoshida H. Global cognition and 8-year survival among Japanese community-dwelling older adults. Int J Geriatr Psychiatry. 2013;28(8):841-849.

10. Park MH, Kwon DY, Jung JM, Han C, Jo I, Jo SA. Mini-mental status examination as predictors of mortality in the elderly. Acta Psychiatr Scand. 2013;127(4):298-304.

11. World Health Organization. International Statistical Classification of Diseases and Related Health Problems, 10th Revision. Geneva: World Health Organization; 2010. Available from: http://apps.who.int/ classifications/icd10/browse/2010/en. Accessed December 10, 2013.

12. Borjesson-Hanson A, Gustafson D, Skoog I. Five-year mortality in relation to dementia and cognitive function in 95-year-olds. Neurology. 2007;69(22):2069-2075.

13. Schultz-Larsen K, Rahmanfard N, Kreiner S, Avlund K, Holst C. Cognitive impairment as assessed by a short form of MMSE was predictive of mortality. J Clin Epidemiol. 2008;61(12):1227-1233.
14. Strandberg TE, Pitkala KH, Tilvis RS. Predictors of mortality in homedwelling patients with cardiovascular disease aged 75 and older. J Am Geriatr Soc. 2009;57(2):279-284.

15. Schaller F, Sidelnikov E, Theiler R, et al. Mild to moderate cognitive impairment is a major risk factor for mortality and nursing home admission in the first year after hip fracture. Bone. 2012;51(3):347-352.

16. Carcaillon L, Peres K, Pere JJ, Helmer C, Orgogozo JM, Dartigues JF. Fast cognitive decline at the time of dementia diagnosis: A major prognostic factor for survival in the community. Dement Geriatr Disord. 2007;23(6):439-445.

17. Rizzuto D, Bellocco R, Kivipelto M, Clerici F, Wimo A, Fratiglioni L. Dementia after age 75 : survival in different severity stages and years of life lost. Curr Alzheimer Res. 2012;9(7):795-800.

18. Xie J, Brayne C, Matthews FE; Medical Research Council Cognitive Function and Ageing Study collaborators. Survival times in people with dementia: analysis from population based cohort study with 14 year follow-up. BMJ. 2008;336(7638):258-262.

19. Nasreddine ZS, Phillips NA, Bedirian V, et al. The Montreal cognitive assessment, MoCA: A brief screening tool for mild cognitive impairment. J Am Geriatr Soc. 2005;53(4):695-699.

20. Tariq SH, Tumosa N, Chibnall JT, Perry MH 3rd, Morley JE. Comparison of the Saint Louis University mental status examination and mini-mental state examination for detecting dementia and mild neurocognitive disorder - a pilot study. Am J Geriatr Psychiatry. 2006;14(11):900-910.

21. Spencer JR, Wendell CR, Giggey PP, et al. Psychometric limitations of the mini-mental state examination among nondemented older adults: An evaluation of neurocognitive and magnetic resonance imaging correlates. Exp Aging Res. 2013;39(4):382-397.
Clinical Interventions in Aging

\section{Publish your work in this journal}

Clinical Interventions in Aging is an international, peer-reviewed journal focusing on evidence-based reports on the value or lack thereof of treatments intended to prevent or delay the onset of maladaptive correlates of aging in human beings. This journal is indexed on PubMed Central, MedLine,

\section{Dovepress}

CAS, Scopus and the Elsevier Bibliographic databases. The manuscript management system is completely online and includes a very quick and fair peer-review system, which is all easy to use. Visit http://www.dovepress. com/testimonials.php to read real quotes from published authors. 